\title{
8 Herausforderungen an Interoperabilität im Arzneimittelbereich
}

\author{
Christine Haas
}

Jährlich werden ca. 15\% aller Gesundheitsausgaben für Arzneimittel aufgewendet [ABDA, 2013]. Jedem in der gesetzlichen Krankenversicherung Versicherten werden jährlich durchschnittlich ca. 14 Arzneimittel verordnet, mit durchschnittlich neun Verordnungen jährlich verlässt er die Arztpraxis [ABDA, 2013].

Der Arzt kann für seine Verordnungen aus 57.000 apotheken- oder verschreibungspflichtigen Humanarzneimitteln auswählen (ohne die verschiedenen Packungsgrößen und versorgungsrechtlichen Rahmenbedingungen zu berücksichtigen), ca. 27.000 davon sind verschreibungspflichtig [DIMDI, 2014]. Pharmazeutische Unternehmen und Zulassungsbehörden in Deutschland sind intensiv damit beschäftigt, die Zulassungsunterlagen von über 100.000 verkehrsfähigen Humanarzneimitteln (inklusive der freiverkäuflichen Produkte) auf dem aktuellen Stand zu halten und damit eine sichere und hochwertige Bereitstellung zu gewährleisten [PharmNet.Bund, 2013].

Ohne Unterstützung durch aktuelle und auf die Bedürfnisse zugeschnittene Softwarelösungen ist es für den Arzt oder Apotheker praktisch unmöglich, sich in dieser Datenflut täglich zu orientieren und das für den Patienten geeignete Medikament auszuwählen.

\subsection{Schauplätze für Arzneimittelinformation}

\subsubsection{Die Fachinformation als rechtlich verbindliche Informationsquelle}

Die Fachinformation fasst den Stand des Wissens zu einem Arzneimittel zusammen. Sie wird regelmäßig durch die pharmazeutische Industrie fortge- 
B Der fachliche Hintergrund: Terminologien und Ordnungssysteme in der

Patientenversorgung, medizinischen Forschung und Gesundheitswirtschaft

schrieben, z.B. werden neu aufgetretene Nebenwirkungen ergänzt oder zusätzliche Warnhinweise oder Kontraindikationen aufgenommen. Die amtlichen Texte, also Fach- und Gebrauchsinformationen, werden durch die Arzneimittelzulassungsbehörde geprüft und der Öffentlichkeit zugänglich gemacht [PharmNet.Bund, 2013]. Für den Arzt oder Apotheker sind sie die rechtlich verbindliche Informationsquelle, an der sich der bestimmungsgemäße Gebrauch orientiert und die ihn auch in seiner Verordnungsentscheidung rechtlich absichert.

Der Aufbau der Fachinformation ist in Richtlinie 2001/83/EG Artikel 11 [EP/EC, 2001] sowie im Arzneimittelgesetz $₫ 11 \mathrm{a}$ [AMG, 1976/2013] vorgegeben und wird durch die Leitlinie zur Zusammenfassung der Produktmerkmale (Guideline on Summary of Product Characteristics, SmPC) [EC, 2009] spezifiziert. Die Dokumentvorlagen werden als „europäische Gesamtlösung“ durch die bei der European Medicines Agency (EMA) angesiedelte Arbeitsgruppe „Quality Review of Documents“ (QRD) kontinuierlich weiterentwickelt und als QRD-Templates [EMA, 1995-2014a] veröffentlicht, damit die pharmazeutischen Unternehmen einen einheitlichen Aufbau der Dokumente in vorgegebenen Kapiteln umsetzen und Arzt und Apotheker sich damit schnell innerhalb der jeweiligen Fachinformation orientieren können. Die Umsetzung ist für pharmazeutische Unternehmen jedoch nicht verpflichtend, sodass die Struktur der Fachinformation dem Format entspricht, zu dem sie eingereicht oder ggf. erneuert wurde.

Da für die Fachinformation nur an ganz wenigen Stellen auch inhaltliche (semantische) Strukturvorgaben gemacht werden, wie beispielsweise bei der Darreichungsform, der Anwendungsart und den Lagerungshinweisen, ist die Fachinformation nicht geeignet, wesentliche Informationen in strukturierter Form und damit technisch auswertbar zu transportieren.

Im Rahmen des Projektes PIM (Product Information Management) konnten die amtlichen Texte bei der EMA in strukturierter Form übermittelt werden, d.h. die wenigen semantischen Teile und Freitextinformationen wurden jeweils strukturiert den einzelnen Kapiteln zugeordnet. Dieses Projekt wurde jedoch im Frühjahr 2011 von der EMA nicht weitergeführt [EMA, 2011]. Eine darauf folgende weitere vielversprechende Initiative der EMA in 2011 zu eSPC (electronic Summary of Product Characteristics) wurde ebenfalls bisher nicht weitergeführt [SIDI, 2011].

Richtungsweisend für die technische Auswertung der Fachinformation ist die Vorgehensweise der US Food and Drug Administration (FDA). Im Rahmen der Zulassung müssen die wesentlichen Informationen zu einem Arzneimittel in strukturierter Form (Structured Product Labelling, SPL im HL7 Format) übermittelt werden. Die Behörde gibt dafür ein elektronisches Format und eine Vielzahl von semantischen Elementen u.a. in SNOMED CT vor [FDA, 2014]. Diese technisch aufbereitete Form ermöglicht eine formale Validierung bereits zum Zeit- 
punkt der Einreichung und eine Verknüpfung mit behördlichen Datenressourcen.

Eine weitergehende (strukturelle und semantische) Strukturierung der Fachinformation und deren Bereitstellung in einem technisch auslesbaren Format ist eine wesentliche Forderung des 7+1-Punkte-Plans der Maßnahme $13 \mathrm{im}$ Rahmen des Aktionsplans Arzneimitteltherapiesicherheit (AMTS) des Bundesministeriums für Gesundheit (BMG) [BMG, 2013b], [AKd̈̈, 2009]. Dies kann jedoch nur im europäischen Konsens gelingen.

\subsubsection{Arzneimittelinformationen in der Arzneimittelversorgung}

Eine Schlüsselfunktion in der Arzneimittelversorgung kommt der Informationsstelle für Arzneispezialitäten (IFA) [IFA GmbH, 2014] zu, die die Arzneimittellogistik und -warenwirtschaft durch Vergabe der Pharmazentralnummer (PZN) bzw. der Pharmacy Product Number (PPN) steuert. Dem ABDATA Pharma-Daten-Service [WuV, 2014] obliegt die Sisyphusaufgabe, diesen Arzneimitteldaten die versorgungsrechtlichen Rahmenbedingungen nach $\$ 129$ Abs. 2 SGB V zuzuordnen [GKV-Spitzenverband, 2014] und damit die Arzneimittelerstattung „lebbar“ zu machen. Unter anderem werden die Arzneimittel den Rabattverträgen der einzelnen Partner zugeordnet, die Festbeträge und Festbetragsgruppen eingepflegt, Wirkstoff- und Darreichungsform-bezogen Aut-Idem-Regeln hinterlegt und der ATC-Kode ergänzt, der beispielsweise die Berechnung der Daten für den Morbi-RSA steuert. Ein neuer Schauplatz ist auch das ARMIN-Projekt zur wirkstoffbezogenen Verordnung, das in 2016 an den Start gehen soll [ARMIN, 2014]. Die Umsetzung der einzelnen versorgungsrechtlichen Anforderungen erfordert eine „maßgeschneiderte“ Anpassung der strukturierten Daten.

Ausgehend von dem Basisdatensatz der IFA oder der ABDATA und den Fachund Gebrauchsinformationen als amtliche und damit rechtsverbindliche Texte bereiten die verschiedenen Softwareanbieter im Arzneimittelbereich die Informationen zu den Arzneimitteln ihres Portfolios angepasst an die Anforderungen ihrer Informationssysteme inhaltlich und datentechnisch auf. Dies schließt ein, dass strukturierte Informationen des Basisdatensatzes für die Zwecke der Verordnungsunterstützung geändert werden. Je nach Ausrichtung des Softwareunternehmens werden bei der Bereitstellung der Arzneimittelinformationen internationale Kodierungen berücksichtigt, ggf. erweitert oder Eigenlösungen entwickelt.

\subsection{3 eMedikation und Einheitlicher Medikationsplan}

Mit der Verabschiedung der Richtlinie 2011/24/EU über die Ausübung der Patientenrechte in der grenzüberschreitenden Gesundheitsversorgung (Patien- 
B Der fachliche Hintergrund: Terminologien und Ordnungssysteme in der

Patientenversorgung, medizinischen Forschung und Gesundheitswirtschaft

ten-Mobilitäts-Richtlinie) [EP/EC, 2011] und der Durchführungsrichtlinie 2012/52/EU [EC, 2012b] wurden die Informationsbausteine festgelegt, die auf einem europäischen Arzneimittelrezept stehen müssen. Erklärtes Ziel des Aktionsplans für elektronische Gesundheitsdienste 2012-2020 [EC, 2012a] der EUKommission ist es, eHealth in Europa auszubauen und den Weg für einen elektronischen Austausch von Patienten- und Medikationsdaten zu ebnen. Die Rahmenbedingungen für eine elektronisch unterstützte, grenzüberschreitende Arzneimittelversorgung (eRezept und Patientenakte) wurden in einem millionenschweren und sehr arbeitsintensiven europäischen Pilotprojekt, European Patients Smart Open Services (epSOS) [epSOS, 2008-2014], analysiert und die Machbarkeit des grenzüberschreitenden und sprachunabhängigen Austausches von Patienten- und Medikationsdaten getestet.

Ein erster Schritt in Deutschland auf dem Weg zum elektronischen Austausch von Medikationsdaten könnte sein, einen einheitlichen Medikationsplan für den Patienten einzuführen, der alle Medikamente, Behandlungsgründe, Dosierungen und Einnahmehinweise auflistet. Im Rahmen des Aktionsplans Arzneimitteltherapiesicherheit (AMTS) wurde eine Spezifikation [AKdÄ, 2013] für den Bundes-Medikationsplan entwickelt, die bis 2015 in Modellprojekten getestet und evaluiert werden wird (Maßnahmen 21 bis 23 des aktuellen Aktionsplans 2013-2015) [BMG, 2013a]. Die Herausforderung besteht hier in dem Spagat zwischen „so einfach wie möglich“, um die elektronische Erfassung für den Arzt und Apotheker in den Praxis- und Offizinalltag zu integrieren, und „so sicher wie möglich“, um einen systemübergreifenden korrekten Datentransfer oder auch die Erfassung komplexer Anforderungen beispielsweise bei der Darstellung der Dosierung und dem Behandlungsgrund (zum einen aus der Sicht des Patienten und zum anderen aus der Sicht der Fachkreise) abbilden zu können. Bei der Umsetzung in die Fläche dürfen auch haftungsrechtliche Fragen nicht außer Acht gelassen werden. Der Brückenschlag zu eMedikation und Datenaustausch nach Europa wird hier nur gelingen, wenn die in Europa konsentierten Datenelemente bei diesem Projekt berücksichtigt werden.

\subsubsection{Arzneimittelinformationen und Patientendaten zur Entscheidungs- unterstützung für die Verordnung}

Clinical Decision Support Systeme, die den Arzt bei der Auswahl des richtigen Arzneimittels für den individuellen Patienten unterstützen sollen, funktionieren prinzipiell so, dass strukturierte Datenelemente zum Patienten aus der Patientenakte (Alter, Geschlecht, Diagnosen, Laborwerte) mit strukturierten Datenelementen (Anwendungsgebiete, Dosierung, Kontraindikationen) zum Arzneimittel aus verschiedenen Quellen regelbasiert abgeglichen werden (s. Abb. 1). Innerhalb einer Praxisverordnungssoftware (PVS) oder eines Krankenhaus-Informationssystems (KIS) werden die zugrundeliegenden Quellen 


\section{Arzneimitteldaten}

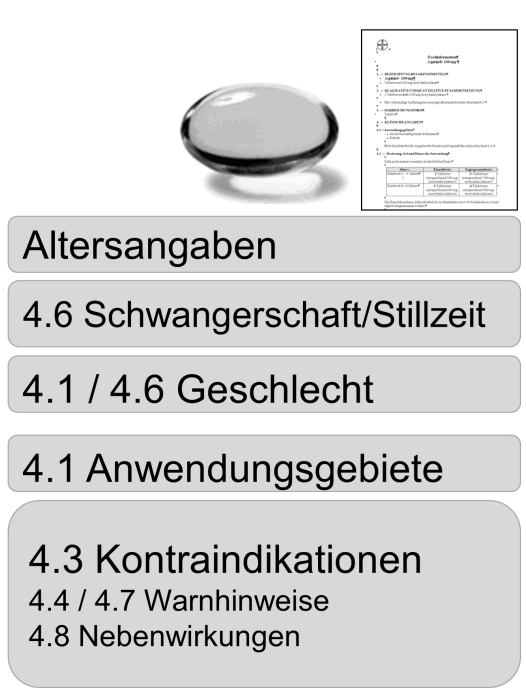

\section{Patientendaten}

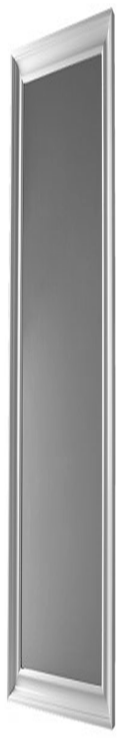

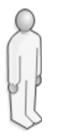

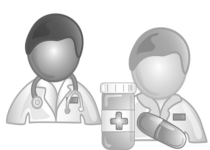

Alter

Lebenssituation

Geschlecht

Behandlungsgrund

Allergien

Laborparameter

Niereninsuffizienz

Abb. 1 Arbeitsweise von Clinical Decision Support Systemen

durch den Softwareanbieter entsprechend strukturell aufbereitet und Regeln festgelegt.

Nicht immer können Situationen im Behandlungsalltag durch Angaben in der Fachinformation abgedeckt werden (wie Empfehlungen für besondere Patientengruppen wie z.B. ältere Patienten, Empfehlungen zur Anwendung in der Pädiatrie oder Aussagen zur Anwendung in der Stillzeit oder Multimedikation). Die Softwarehersteller werten dafür mit erheblichem Aufwand die einschlägige Fachliteratur aus oder bedienen sich Sekundärquellen. Ein Beispiel dafür sind die ABDA-Wirkstoffdossiers, die den Arzt und Apotheker bei der Beratung in speziellen Situationen unterstützen. Das Praxis- und Forschungswissen jenseits der Fachinformation liegt in vielen Quellen oft bereits vor (z.B. Spezial- und Forschungsdatenbanken, Cochrane). Eine Festlegung auf sektorübergreifende und international kompatible Referenzterminologien (z.B. zur Verschlagwortung von Wirkstoffen und Patientengruppen) könnte eine zeitnahe Erschließung von entsprechend verschlagworteten dezentral vorliegenden Quellen und eine Vernetzung des Therapiewissens unterstützen. Dies wurde auch im Rahmen des 7+1-Punkte-Plans des Aktionsplans AMTS 2008-2010 postuliert [BMG, 2013b], [AKd ̈̈, 2009]. 


\subsection{Nationale und internationale Referenzstrukturen}

\subsubsection{Arzneimittelidentifikatoren}

Die Pharmazentralnummer (PZN) bzw. die Pharmacy Product Number (PPN) ist der Schlüssel nach $\mathbb{3} 300$ Abs. 1 Satz 1 Nr. 1 des Sozialgesetzbuches V. Sie identifiziert eine Arzneimittelpackung, die in der Apotheke abgegeben wird und ist damit das Kernelement für die Arzneimittelversorgung. Für Arzneimittel, die außerhalb der öffentlichen Apotheke vertrieben werden, werden oft keine kostenpflichtigen Pharmazentralnummern beantragt. Gemäß der \$\$ 10 und 11a des Arzneimittelgesetzes (AMC) muss eine Arzneimittelpackung zudem mit der Zulassungsnummer (ZNR) gekennzeichnet sein. In Deutschland wird die ZNR allen Packungsgrößen eines Arzneimittels zugeordnet, für zentral in Europa zugelassene, innovative Arzneimittel wird eine ZNR einer Packungsgröße - analog zur PZN - zugeordnet. Bei einem Pilotprojekt zwischen DIMDI und ABDATA ist es nur zu einem Bruchteil gelungen, die behördlichen Daten [PharmNet.Bund, 2013] und die Versorgungsdaten [DIMDI, 2014] eindeutig einander zuzuordnen [Haas et al., 2007]. Als Ergebnis dieser wenig fruchtbaren Bemühungen erfasst nun die IFA auch die ZNR verpflichtend, sodass eine Zuordnung möglich ist (s. Abb. 2).

Im Juli 2012 waren die pharmazeutischen Unternehmen in Europa aufgefordert, Kerndaten für die in Europa auf dem Markt befindlichen Arzneimittel

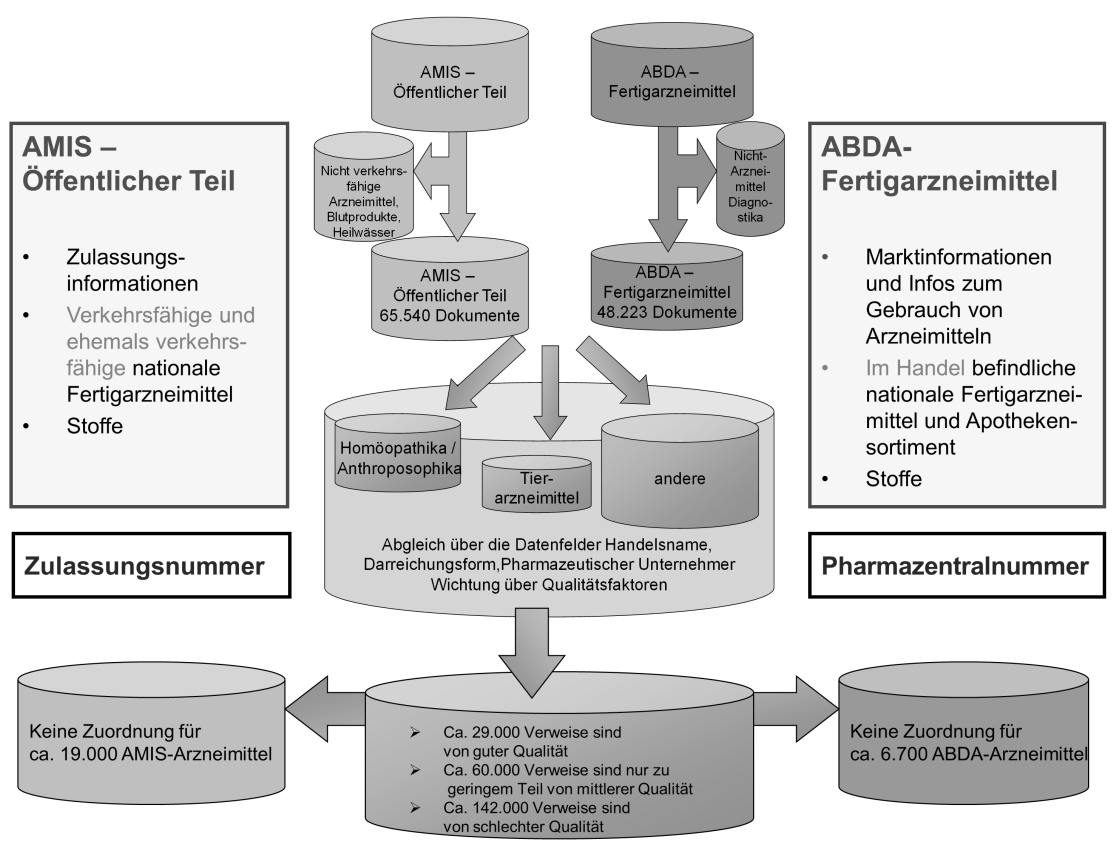

Abb. 2 Zuordnung der Zulassungsdaten aus AMIS Öffentlicher Teil zu den Versorgungsdaten aus ABDA Fertigarzneimittel 
im Rahmen der Pharmacovigilance Legislation 2010 [EP/EC, 2010] in elektronischer Form zu übermitteln [EMA, 1995-2014b]. Diese Kerndaten sind international als ISO Norm 11615 standardisiert [ISO, 2012d]. Jedem neu gemeldeten Arzneimittel wird ein Medicinal Product Identifier (MPID) für jede Zulassungsnummer und ein Medicinal Product Package Identifier (PCID) für jede Packungsgröße zugewiesen. Diese beiden Identifikatoren sind prinzipiell geeignet, den arzneimittelbezogenen Informationsaustausch in Europa zu ermöglichen. Die pharmazeutischen Unternehmen sind derzeit damit beschäftigt, die bereits gemeldeten Datensätze zu aktualisieren und auf ein neues Datenmodell anzupassen. Fragen zu Maintenance und Validierung der gemeldeten Daten sind jedoch (noch) nicht gelöst, sodass die erfassten Daten zum aktuellen Stand nur informativen Charakter haben können.

\subsubsection{Stoffidentifikatoren}

Die Arzneimittelzulassungsbehörde kommt erstmals mit wirkstoffbezogenen Daten zum Zeitpunkt der Anmeldung einer Klinischen Prüfung in Berührung. $\mathrm{Zu}$ diesem Zeitpunkt wird dem Wirkstoff eine Arzneistoffkatalognummer (ASK-Nummer) zugewiesen, die die behördeninternen Verfahren im gesamten Life-Cycle begleitet. Die ASK-Nummer muss im Zulassungsantrag angegeben werden, die zugehörige Vorzugsbezeichnung ist gemäß $\$ 10$ Abs. 6 Nr. 1 Satz 2 AMG auf der Packung und in den amtlichen Texten anzugeben. Die Vorzugsbezeichnung orientiert sich üblicherweise am International Nonproprietary Name der WHO (INN). Im AMIS-Stoffkatalog wird der Vorzugsbezeichnung auch der Klinische Prüfcode und - wenn (bei chemisch definierten Stoffen) vorhanden - auch die Identifikationsnummer des Chemical Abstract Service (CAS-Nummer) zugeordnet. Der Stoffkatalog deckt damit alle Stoffe von Arzneimitteln ab, die in Deutschland zugelassen sind oder waren. Er hat jedoch ausschließlich Bedeutung im Geltungsbereich des AMG.

Im epSOS-Projekt wurde die 5. Stufe der Anatomisch-Therapeutisch-Chemischen Klassifikation (ATC-Kode) zur Übermittlung von Wirkstoffinformationen vorgeschlagen. Im Rahmen der Evaluierung musste jedoch festgestellt werden, dass der ATC-Kode der WHO bei Arzneimitteln, die Wirkstoffkombinationen enthalten, an Grenzen stößt. Aufgrund seiner Zielsetzung enthält der ATC-Kode der WHO ausschließlich chemisch definierte Stoffe. Weiterhin wurden Unsicherheiten hinsichtlich der „Übersetzung“ der Dosierungsangaben zum jeweiligen Wirkstoff identifiziert, die sich nicht einheitlich auf den wirksamkeitsbestimmenden Anteil oder den pharmazeutischen Stoff (beispielsweise mehrere Salze mit unterschiedlichem Molekulargewicht) beziehen. Beim Austausch eines Präparates müsste eine mögliche Über- oder Unterdosierung durch diese Unschärfe in Kauf genommen werden. In Deutschland ist das wissenschaftliche Institut der AOK für die Bereitstellung der ATC-Klassifikation für Deutschland zuständig. Diese ergänzt den ATC-WHO um die 
B Der fachliche Hintergrund: Terminologien und Ordnungssysteme in der

Patientenversorgung, medizinischen Forschung und Gesundheitswirtschaft

„Bedürfnisse“ des deutschen Gesundheitswesens und umfasst zusätzlich zu chemisch definierten Arzneistoffen unter anderem auch pflanzliche Stoffe. Eine Nutzung ist nur im Rahmen des $₫ 73$ Sozialgesetzbuch V vorgesehen. Da Wirkstoffe zum Teil mehreren Anwendungsgebieten zugeordnet werden, ist der ATC-Code als eindeutiger wirkstoffbezogener Schlüssel nicht geeignet.

Die verschiedenen Stoffbezeichnungen und Kodierungen (CAS-Nummer, INNName, ATC-Kode, ASK-Nummer) werden üblicherweise auch in den Stoffkatalogen referenziert, die in den einzelnen Softwaresystemen zu Arzneimitteln hinterlegt sind. Da die Regeln zur Auswahl des richtigen Arzneimittels bei der Verordnung weitgehend stoffbezogen definiert werden, ist der Stoffkatalog das „Herzstück“ jedes Computerised Physician Order Entry (CPOE) Systems POE und Clinical Decision Support Systems (CDSS). Die Struktur und Systematik jedes Stoffkataloges hängt damit individuell von den Fragestellungen ab, die stoffbasiert „bedient“ werden sollen. Die ABDATA hat in den letzten Monaten intensiv den ABDA-Stoffkatalog überarbeitet und für die Zwecke der stoffbezogenen Verordnung im Rahmen des ARMIN-Projekts [ARMIN, 2014] angepasst. Seit Januar 2014 kann der Stoffkatalog kostenfrei in elektronischer Form bezogen werden [Pflugmann, 2013]. Wie die ASK-Nummer hat der ABDAStoffkatalog keine internationale Bedeutung.

Im Rahmen der ISO-IDMP-Aktivitäten, die rund um die Pharmacovigilance Legislation [EMA, 1995-2014b] betrieben wurden, wurde nicht nur der Grundstein für ein internationales Arzneimittelregister (ISO Norm 11615) [ISO, 2012d], sondern auch für ein internationales Stoffregister (ISO Norm 11238) [ISO, 2012a] gelegt. Eine international konsentierte Datenstruktur soll es ermöglichen, zu einem Stoff alle Informationen, die im Zulassungsbereich benötigt werden, zu erfassen, einen international gültigen Identifier zu vergeben und über eine zentrale Struktur zu vernetzen. Eine Expertengruppe aus Behördenvertretern arbeitet intensiv an der Implementierung des Global Ingredient Archival System GInAS [GInAS, 2014]. Der Pilotbetrieb soll in 2014 mit einem Datenpool der beteiligten Behörden (unter anderem der Stoffdaten des BfArM und deren ASKNummer) aufgenommen werden. In einem Verbändetreffen in 2013 im Bundesinstitut für Arzneimittel und Medizinprodukte (BfArM) hat die pharmazeutische Industrie Unterstützung bekundet, die erforderlichen Informationen zu Stoffen im Sinne eines „Substance Master Files“ an einer zentralen Stelle bereitzustellen und so zukünftig nur einmal weltweit im Zulassungswesen referenzieren zu müssen. GInAS soll Daten enthalten, die einen Wirkstoff charakterisieren wie Aufbau, Nomenklatur und Synonyme oder chemische Eigenschaften. In einem vertraulichen Bereich sollen weiter auch Informationen zu dessen Herstellungsverfahren, zu Abbauprodukten und Verunreinigungen gepflegt werden können. GInAS könnte also in Zukunft ein „Metaregister“ für Stoffinformationen werden, auf das andere Stoffregister im Arzneimittelbereich referenzieren können. 


\subsubsection{Darreichungsform und Anwendungsart}

In der Fachinformation ist die Nutzung der Standard Terms des European Directorate for the Quality of Medicines and Healthcare (EDQM) [EDQM, 20082014] zu Darreichungsform, Anwendungsart und Behältnis vorgeschrieben. Jede Arzneimittelpackung hat diese strukturierten Elemente zur Bezeichnung der Darreichungsform und Anwendungsart gemäß $₫ 10$ AMG aufgedruckt. Die Standard Terms haben eine enge Verknüpfung mit der Weiterentwicklung des Europäischen Arzneibuches (European Pharmacopoea) und der entsprechenden Monographien, die eine Darreichungsform oder Anwendungsart beschreiben. Die Begrifflichkeiten sind daher genau definiert und in alle europäischen und zum Teil in weitere Sprachen übersetzt. Die Mitarbeiter der EDQM sind an einem weiteren Standard im ISO-IDMP-Standardpaket „Data elements and structures for the unique identification and exchange of regulated information on pharmaceutical dose forms, units of presentation, routes of administration and packaging“ (ISO 11239) [ISO, 2012b] maßgeblich beteiligt, sodass diese in internationale Referenzstrukturen einfließen werden.

\subsubsection{Maßeinheiten und Dosierung}

International hat sich in überregionalen Projekten im Gesundheitswesen die Darstellung von Einheiten mittels UCUM (The Unified Code for Units of Measure), entwickelt durch das Regenstrief Institute, durchgesetzt [Schadow und McDonald, 1999-2013]. UCUM ist auch die Grundlage für die ISO-Norm 11240 des ISO-IDMP-Standardpaketes [ISO, 2012C].

Richtungsweisend zur Darstellung von Dosierungen ist das Dosage Syntax Abstract Model des NHS, welches auf HL7 basiert [Glover und James, 2005]. Das Modell wurde in 2012 in vereinfachter Form bei ISO eingereicht (PDTS 17251.3) [ISO, 2013].

\subsubsection{Indikationen, Nebenwirkungen}

Im ISO-IDMP-Projekt wurde die Suche nach einer international zu nutzenden Terminologie zur Beschreibung von Indikationen, Nebenwirkungen und Kontraindikationen (s. Abb. 3) ausgeklammert, weil hier international kein Konsens zu finden war. In Europa soll bei der Einreichung von Arzneimitteldaten im Rahmen der Pharmacovigilance Legislation (XEVMPD) [EMA, 1995-2014b] die Indikation mit MedDRA (Medical Dictionary for Regulatory Activities) [MedDRA] kodiert werden.

In den USA wird die „Medical Condition“ des Structured Product Labelling (10) mittels SNOMED CT [IHTSDO, 2014] kodiert, die Indication Category soll durch LOINC-Codes [Regenstrief-Institute, 1994-2014] strukturiert erfasst werden. 
B Der fachliche Hintergrund: Terminologien und Ordnungssysteme in der Patientenversorgung, medizinischen Forschung und Gesundheitswirtschaft

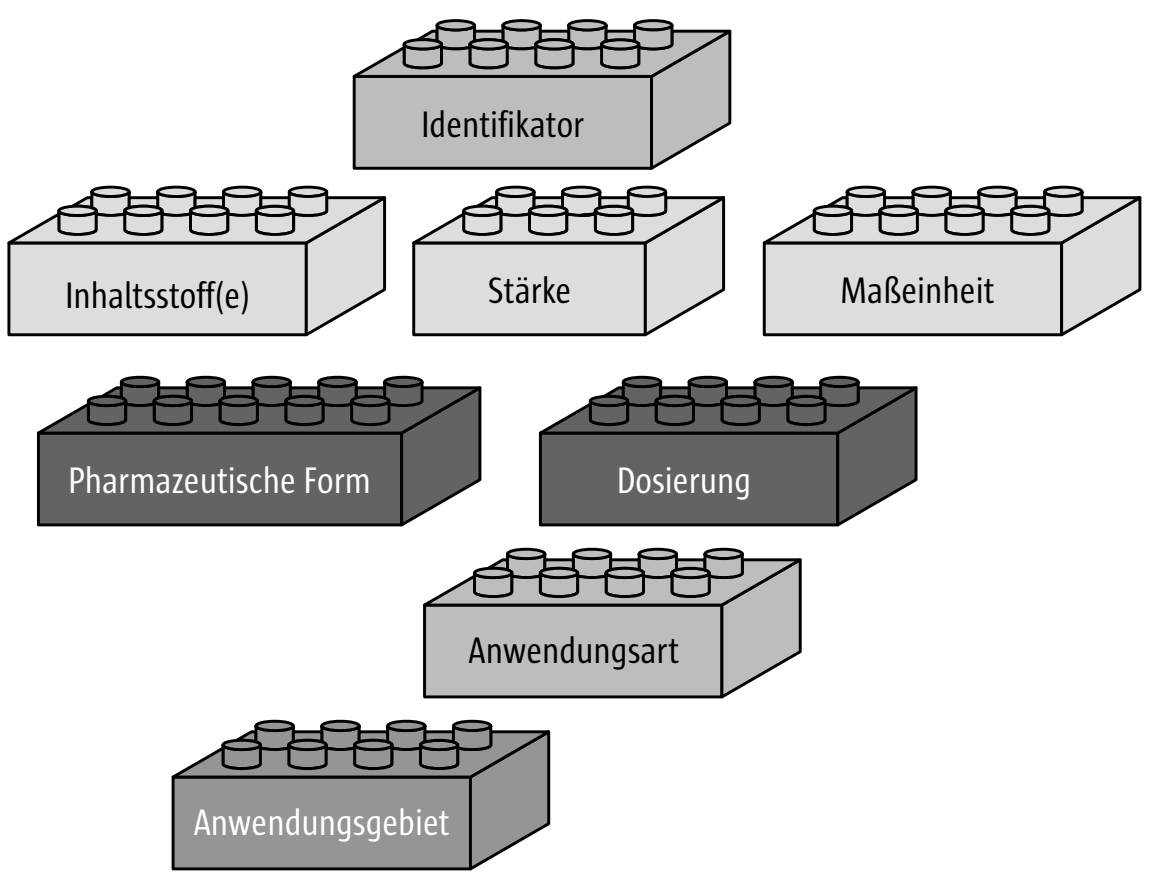

Abb. 3 Strukturierte Elemente zur Beschreibung von Arzneimittel und Medikation

Die Medizinische Medien Informations $\mathrm{GmbH}$ (MMI) bereitet im Rahmen des DIMAS-Projekts Informationen zu Indikation, Nebenwirkungen und Kontraindikationen mithilfe des ICD-10-GM bzw. des erweiterten Synonymverzeichnisses der Alpha-ID auf [Brause und Christ, 20o9], [Schulte van Werde et al., 2008].

Die Belastbarkeit der erfassten Daten ist Gegenstand regelmäßiger Diskussionen zur Datenqualität und Datenkonsistenz der erhobenen Daten, denn die Auswahl der korrekten Kodes bedarf eines intensiven Trainings und terminologischer Expertise sowie einer regelmäßigen Praxis im Umgang mit den jeweiligen Terminologiesystemen.

\subsection{Voraussetzung für Interoperabilität zu Arzneimittel und Medikation}

Wie kann vermieden werden, dass ein Patient dadurch verunsichert wird, dass er auf seiner Arzneimittelpackung andere Bezeichnungen vorfindet, als auf den Angaben seines vom Arzt oder Apotheker ausgestellten Medikationsplans? Wie kann ein Arzt bei der Verordnung aus seiner Praxissoftware heraus auch die jeweils aktuelle amtliche Fachinformation oder den neuesten Rote-HandBrief angezeigt bekommen? 
Dies ist nur möglich, wenn den unterschiedlichen elektronischen Quellen Referenzstrukturen hinterlegt sind, über die sie sich über Softwaresysteme und über Sektoren hinweg austauschen können.

Als Vorbild kann RXNORM der National Library of Medicines (NLM) [NIH, 2005-2014] dienen. Hier arbeiten Arzneimittelbehörde, Forschungsinstitute und Softwarehersteller in den USA zusammen, ein Referenzdatenset für Arzneimittel bereitzustellen, das die o.g. wesentlichen Kernelemente zur Beschreibung von Arzneimitteln und Identifikatoren umfasst. Dieses Referenzdatenset dient als „Datendrehscheibe“, um eine technologieunabhängige Interoperabilität und Kommunikation zwischen den verschiedenen elektronischen Systemen zu ermöglichen und damit Quellen einfacher zu erschließen und Wissen zu vernetzen.

Wie oben beschrieben, gibt es viele unterschiedliche Beteiligte, die Arzneimitteldaten in elektronischer Form bereitstellen. Diese sind jeweils eng an die jeweiligen Rahmenbedingungen gebunden und haben einen unterschiedlichen Fokus hinsichtlich der Präsentation ihrer Daten. Voraussetzung zur Etablierung eines solchen „Basisdatensatzes“ ist daher, unabhängige und neutrale Strukturen für eine fachliche und technische Plattform zu etablieren, auf der sich die verschiedenen Player zusammenfinden können.

Die Schaffung dieser Arzneimittel-Infrastruktur kann nur gelingen, wenn folgende Kriterien berücksichtigt werden:

Unabhängigkeit: Ein breiter Konsens bei allen Akteuren (Zulassung, Versorgung, Forschung) kann nur geschaffen werden, wenn die Referenzstrukturen sektorenübergreifend und transparent von allen Beteiligten gemeinsam weiterentwickelt werden und medizinische, ökonomische, technologische und Public-Health-Aspekte berücksichtigen.

Transparenz: Die Konsensfindung und sektorenübergreifende Akzeptanz sollte in einem offenen Vorschlagsverfahren und einem transparenten Entscheidungsprozess erfolgen. Zusätzlich zur fachlichen Arbeit muss die technische Infrastruktur bereitgestellt werden, um die Referenzstrukturen in der Fläche elektronisch verbreitbar und nutzbar zu machen. Vorhandene Strukturen wie z.B. die Koordinierungsgremien zu Terminologien im DIMDI oder auch von DIN oder dem Interoperabilitätsforum sollten genutzt werden.

Verlässlichkeit: Die Datendrehscheibe muss so gestaltet sein, dass sie eine kontinuierliche Weiterentwicklung und Planung für alle Beteiligten ermöglicht. Dies betrifft nicht nur die Bereitstellung von Referenzterminologien, die quantitativ alle Systeme „durchdringen“, sondern auch die Bereitstellung von Schnittstellen und Datenformaten, über die die Daten zwischen den jeweiligen Softwaresystemen transportiert werden. In diesem Zusammenhang spielen auch Fragen der Nutzung von Lizenzen für Terminologiesysteme eine Rolle, die auf nationaler Ebene gelöst werden müssen. Die Verlässlichkeit sollte auch 
B Der fachliche Hintergrund: Terminologien und Ordnungssysteme in der

Patientenversorgung, medizinischen Forschung und Gesundheitswirtschaft

politisch untermauert werden, indem diese in rechtlichen Rahmenbedingungen umgesetzt wird (z.B. im Arzneimittelgesetz oder Sozialgesetzbuch V wird festgelegt, dass entsprechende Strukturen zu schaffen und auch an die Referenzplattform zu übergeben sind).

Nachhaltigkeit: Änderungen von Datenstrukturen und -inhalten bedeuten für alle Beteiligten einen erheblichen Aufwand. Die Bereitschaft, diesen Aufwand zu betreiben, wird davon abhängen, ob die Investitionen für die Softwarehersteller langfristig einen Mehrwert bedeuten. Kurzfristige Insellösungen müssen daher vermieden werden. Bei der Konsensfindung müssen internationale Entwicklungen und Standards berücksichtigt werden. Nationale Weiterentwicklungen sollten auch in den internationalen Standardisierungsprozess entsprechend eingebracht werden. Technische Strukturen müssen so festgelegt werden, dass sie internationalen Standards entsprechen und damit „wiederverwendbar" sind.

Erweiterbarkeit: Referenzstrukturen, die alle oben skizzierten „Schauplätze“abdecken, wird es nicht geben können. Die Referenzstrukturen können dazu dienen, ein Mindestmaß an Interoperabilität zu schaffen. Diese müssen durch die Softwareanbieter für ihre jeweiligen individuellen Angebote erweitert werden können. Die Abwägung zwischen Wettbewerbsrecht und gesellschaftlichem Auftrag für den Patienten ist hier sicherlich ein Balanceakt.

\section{Referenzen}

[ABDA, 2013] Bundesvereinigung Deutscher Apothekerverbände (ABDA) (2013): Die Apotheke - Zahlen Daten Fakten 2013.

[AKdÄ, 2009] AKdÄ. Maßnahme 13. http://www.akdae.de/AMTS/Massnahmen-2008-2009/M13.html Letzter Zugang: 2014-11-09.

[AKdÄ, 2013] AKdÄ. Medikationsplan V2.0. http://www.akdae.de/AMTS/Medikationsplan/index.html Letzter Zugang.

[AMG, 1976/2013] AMG. Gesetz über den Verkehr mit Arzneimitteln (Arzneimittelgesetz - AMG) in der Fassung der Bekanntmachung vom 12. Dezember 2005 (BGBI. I S. 3394), das durch Artikel 1 der Verordnung vom 25. März 2013 (BGBl. I S. 627) geändert worden ist. Im Internet: http://www.gesetze-im-internet.de/ bundesrecht/amg_1976/gesamt.pdf Letzter Zugang: 2014-09-11.

[ARMIN, 2014] ARMIN. ARMIN - Die Arzneimittelinitiative Sachsen-Thüringen. http://www.arzneimittelinitiative.de/grundlagen/ Letzter Zugang: 2014-09-11.

[BMG, 2013a] Bundesministerium für Gesundheit (2013): Aktionsplan 2013-2015 des Bundesministeriums für Gesundheit zur Verbesserung der Arzneimitteltherapiesicherheit in Deutschland

[BMG, 2013b] Bundesministerium für Gesundheit, Haas, C., Menges, K. und Aly, A.F. (2013): Abschlussbericht Maßnahmen 29 bis 31 - Aktionsplan Arzneimitteltherapiesicherheit 2010 bis 2012. https://www. bundesgesundheitsministerium.de/service/publikationen/einzelansicht.html?tx_rsmpublications_pi1_publication]=1930\&tx_rsmpublications_pi1[action]=show\&tx_rsmpublications_pi1[controller]=Publication \&cHash=3f8c9feocc9d5004a7a4926fc84eg939 Letzter Zugang: 2014-09-22.

[Brause, C. und Christ, P., 2009] Brause, C. und Christ, P. Möglichkeiten und Ergebnisse eines elektronischen Fachinformationsvergleichs mit DIMAS. http://www.aminf02009.de/downloads/poster/Poster_Brause. pdf Letzter Zugang: 2014-09-22.

[DIMDI, 2014] DIMDI. ABDA-Fertigarzneimittel (AE00). https://www.dimdi.de/static/de/amg/abda/aeo0. htm_945116164.htm Letzter Zugang: 2014-09-11. 
[EC, 2009] European Commission, Enterprise and Industry Directorate-General (2009): A guideline on summary of product characteristics (SmPC).

[EC, 2012a] Europäische Kommission (2012a): Aktionsplan für elektronische Gesundheitsdienste 2012-2020 innovative Gesundheitsfürsorge im 21. Jahrhundert

[EC, 2012b] EC. Durchführungsrichtlinie 2012/52/EU der Kommission vom 20. Dezember 2012 mit Maßnahmen zur Erleichterung der Anerkennung von in einem anderen Mitgliedstaat ausgestellten ärztlichen Verschreibungen. Im Internet: http://eur-lex.europa.eu/legal-content/DE/TXT/PDF/?uri=CELEX:32012LO 052\&qid=1408810772263\&from=DE Letzter Zugang: 2014-09-22.

[EDQM, 2008-2014] EDQM. Standard Terms for dosage forms, routes of administration and containers. $h t t p: / /$ www.edgm.eu/StandardTerms/Letzter Zugang: 2014-09-22.

[EMA, 1995-2014a] EMA. Product-information templates. http://www.ema.europa.eu/ema/index.jsp?curl= pages/regulation/document_listing/document_listing_000134.jsp Letzter Zugang: 2014-09-11.

[EMA, 1995-2014b] EMA. Reporting requirements for marketing-authorisation holders. http://www.ema. europa.eu/ema/index.jsp?curl=pages/regulation/general/general_content_000594.jsp\&mid= WCob01ac058078fbe1 Letzter Zugang: 2014-09-22.

[EMA, 2011] EMA. European Medicines Agency closes PIM project. $h t t p: / / w w w . e m a . e u r o p a . e u / e m a / i n d e x$. jsp?curl=pages/news_and_events/news/2011/03/news_detail_001233.jsp\&murl=menus/news_and_ events/news_and_events.jsp\&mid=wcob01ac058004d5c1 Letzter Zugang: 2014-09-11.

[EP/EC, 2001] EP/EC. Richtlinie 2001/83/EG des Europäischen Parlaments und des Rates vom 6. November 2001

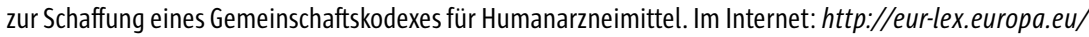
LexUriServ/LexUriServ.do?uri=CONSLEG:2001L0083:20070126:de:PDF Letzter Zugang: 2014-09-11.

[EP/EC, 2010] Europäisches Parlament/Europäischer Rat (2010): Verordnung (EU) Nr. 1235/2010 des Europäischen Parlaments und des Rates vom 15. Dezember 2010 zur Änderung der Verordnung (EG) Nr. 726/2004 zur Festlegung von Gemeinschaftsverfahren für die Genehmigung und Überwachung von Human- und Tierarzneimitteln und zur Errichtung einer Europäischen Arzneimittel-Agentur hinsichtlich der Pharmakovigilanz von Humanarzneimitteln und der Verordnung (EG) Nr. 1394/2007 über Arzneimittel für neuartige Therapien.

[EP/EC, 2011] EP/EC. Richtlinie 2011/24/EU des Europäischen Parlaments und des Rates vom 9. März 2011 über die Ausübung der Patientenrechte in der grenzüberschreitenden Gesundheitsversorgung. Im Internet: http://eur-lex.europa.eu/legal-content/DE/TXT/PDF/?uri=CELEX:32011L0024\&rid=3 Letzter Zugang: 2014-09-11.

[epSOS, 2008-2014] epSOS. European Patients Smart Open Services. http://www.epsos.eu/home.html Letzter Zugang: 2014-09-22.

[FDA, 2014] FDA. Structured Product Labeling Resources. http://www.fda.gov/forindustry/datastandards/ structuredproductlabeling/default.htm Letzter Zugang: 2014-09-11.

[GInAS, 2014] GInAS. Unified Ingredient Information - Open development of a global substance registration tool http://tripod.nih.gov/GInAS/index.htm/ Letzter Zugang: 2014-09-22.

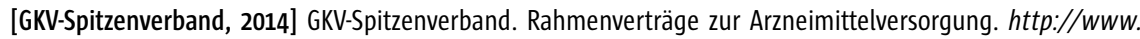
gkv-spitzenverband.de/krankenversicherung/arzneimittel/rahmenvertraege/rahmenvertraege.jsp Letzter Zugang: 2014-09-11.

[Glover, H. und James, I., 2005] Blue Wave Informatics LLP (2005): Final Report of the Dosage Syntax Development Project

[Haas, C. et al., 2007] Haas, C. et al. (2007): Mapping von Arzneimittelverzeichnissen am Beispiel der Datenbanken AMIS-Öffentlicher Teil und ABDA-Fertigarzneimittel Vortrag im Rahmen von: Kongress Medizin und Gesellschaft 2007, Augsburg.

[IFA GmbH, 2014] IFA GmbH. Informationsstelle für Arzneispezialitäten IFA GmbH. http://www.ifaffm.de/de/ home.htm/ Letzter Zugang: 2014-09-11.

[IHTSDO, 2014] IHTSDO. SNOMED CT. http://www.IHTSDO.org/SNOMED-ct Letzter Zugang: 2014-08-12.

[ISO, 2012a] International Organization for Standardization (2012a): ISO 11238:2012 - Health informatics Identification of medicinal products- Data elements and structures for the unique identification and exchange of regulated information on substances. 
B Der fachliche Hintergrund: Terminologien und Ordnungssysteme in der Patientenversorgung, medizinischen Forschung und Gesundheitswirtschaft

[ISO, 2012b] International Organization for Standardization (2012b): ISO 11239:2012 - Health informatics Identification of medicinal products - Data elements and structures for the unique identification and exchange of regulated information on pharmaceutical dose forms, units of presentation, routes of administration and packaging.

[ISO, 2012C] International Organization for Standardization (2012C): ISO 11240:2012 - Health informatics Identification of medicinal products - Data elements and structures for the unique identification and exchange of units of measurement.

[ISO, 2012d] International Organization for Standardization (2012d): ISO 11615:2012 Health informatics - Identification of medicinal products - Data elements and structures for the unique identification and exchange of regulated medicinal product information.

[ISO, 2013] International Organization for Standardization (2013): ISO TC 215/SC N1228 - ISO/PDTS 17251.3 Health Informatics - Requirements for the exchange of structured dose instructions for medicinal products.

[MedDRA, MedDRA. Medical Dictionary for Regulatory Activities. http://www.meddra.org/ Letzter Zugang: 2014-09-22.

[NIH, 2005-2014] NIH. Unified Medical Language System ${ }^{\circledR}\left(\mathrm{UMLS}^{\circledR}\right)$. http://www.nlm.nih.gov/research/ umls/rxnorm/overview.html Letzter Zugang: 2014-09-22.

[Pflugmann, G., 2013] Pflugmann, G. (2013): ABDATA-Stoffkatalog - Verwechslungen ausgeschlossen. Pharmazeutische Zeitung online 50/2013.

[PharmNet.Bund, 2013] PharmNet.Bund. Arzneimittelinformationssystem. http://www.pharmnet-bund.de/ dynamic/de/am-info-system/index.html Letzter Zugang: 2014-09-11.

[Regenstrief-Institute, 1994-2014] Regenstrief-Institute. LOINC ${ }^{\circledR}$ A universal code system for tests, measurements, and observations. http://LOINC.org/Letzter Zugang: 2014-08-12.

[Schadow, G. und McDonald, C.J., 1999-2013] Schadow, G. und McDonald, C.). The Unified Code for Units of Measure. http://unitsofmeasure.org/trac/Letzter Zugang: 2014-09-22.

[Schulte van Werde, M. et al., 2008] Schulte van Werde, M. et al. (2008): Rechnergestützter Vergleich von Fachinformationen mit dem Drug Information Management System (DIMAS). Die pharmazeutische Industrie 70 (2): S. 187.

[SIDI, 2011] SIDI. Video recording of the eSPC Workshop at EMA. http://www.sidi.se/node/32 Letzter Zugang: 2014-09-11.

[WuV, 2014] WuV. ABDATA Pharma-Daten-Service. http://www.wuv-gmbh.de/abdata-pharma-daten-service/ Letzter Zugang: 2014-09-11. 